\title{
Thyroid Hormone Iodination Alteration
}

National Cancer Institute

\section{Source}

National Cancer Institute. Thyroid Hormone lodination Alteration. NCI Thesaurus. Code C40687.

A finding indicating a deficiency or excess in iodination of thyroid hormone. 